\title{
Some Empirical Observations on Diesel Particulate Filter Modeling and Comparison Between Simulations and Experiments
}

Copyright $(92000$ Society of Automotive Engineers, Inc.

\author{
P. Versaevel, H. Colas, C. Rigaudeau, R. Noirot
}

PSA Peugeot-Citroën, La Garenne-Colombes, France

G. C. Koltsakis, A. M. Stamatelos

University of Thessaly, Greece

\begin{abstract}
Comparisons between 1D simulations and experiments on a mini scale SiC filter are presented.

First of all, experiments with regeneration for different loading mass and soot composition enabled us to derive an improved pressure drop correlation. The assumption of constant particulate layer permeability proves unable to predict the influence of the gas temperature on the pressure drop. This discrepancy seems to be linked to the high Knudsen number of the flow in the particulate layer. A new correlation is proposed. This correlation contains four adjustable constants which have been determined on a single experimental run. Without modifying these constants, other cases have been correctly simulated. Obviously, more work is needed to substantiate this approach.
\end{abstract}

In a second step, regenerations with and without additive (Cerium) for two different soot compositions have been simulated and compared with experimental results. Soluble Organic Fraction vaporization has to be taken into account to obtain the right soot mass when regeneration begins. The experimental trend is well captured by numerical simulations.

\section{INTRODUCTION}

There is an increasing need to develop reliable and durable trap systems which meet tough market and legislative standards regarding vehicle performance and fuel economy, as well as trap filtration efficiency. The design of a diesel particulate trap system to fit a specific vehicular application requires significant expenditure, due to the high degree of interaction between the vehicle operation and trap behavior. This interaction may be even more critical in the case of a fuel additive assisted trap system. The assistance of modeling in the design process is already well established.

During the last fifteen years, a significant number of trap system versions has been presented. The vast experience gained during this period, which was accompanied by a significant number of trap failures, led to the conclusion that trap system design must be supported by computer aided engineering. Mathematical models should be able to describe in a physically sound yet practical way the dominating processes in the particulate filter, namely:

- filter back-pressure as function of geometrical and operating parameters for different particulate loadings.

- thermal and chemical processes inside the filter, including heat transfer and particulate oxidation by exhaust oxygen (with or without the use of catalytic aids).

A significant number of modeling efforts in the above directions has appeared in the related literature. Some of the published works have claimed good agreement between measured and predicted results regarding pressure drop as well as regeneration evolution. However, the main difficulty in assessing model accuracy is the uncertainty in the experimental conditions themselves. Laboratory studies with mini scale filters and synthetic soot can be very well controlled but the soot properties are not absolutely representative to the particulate accumulated during real driving. On the other hand, a test with a real filter loaded and regenerated with real exhaust is more realistic, but the operating conditions (particulate loading, flow distribution in the filter, exhaust gas properties) are more difficult to control.

For the above reasons, the validation of diesel filter mathematical models is a quite complicated task and may easily lead to erroneous conclusions. Based on our previous modeling and experimental experience, we present an improved modeling approach, giving special emphasis to the pressure drop model. The pressure drop and regeneration model is validated in synthetic gas bench tests with mini scale traps, which have been loaded with real exhaust under typical vehicle conditions. In this way, we have wellcontrolled experimental conditions and at the same time realistic particulate properties. 


\section{EXPERIMENTS}

\section{APPARATUS}

In order to load and regenerate Diesel particulate filter samples, two benches are used. The first one is an engine test bench devoted to loading. It is equipped with torque and speed closed loop controllers. The second one is devoted to regeneration (see Figure 1). It is made of refractory steel which can be heated up to $1400 \mathrm{~K}$. The reactor is insulated from the outside with a vacuum gap. The inlet gas flows through a heater, which can heat the gas from ambient to $973 \mathrm{~K}$.

Parameters controlled at inlet are mass flow rate, gas temperature and $\mathrm{O}_{2}$ molar fraction.

Five thermocouples are used. Two thermocouples measure the gas temperature upstream and downstream the filter. The filter itself is equipped with three thermocouples: $z=0 \mathrm{~cm}$, $\mathrm{z}=10 \mathrm{~cm}$ and $\mathrm{z}=14 \mathrm{~cm}$.

The pressure drop is continuously measured together with the outlet $\mathrm{CO}, \mathrm{CO}_{2}, \mathrm{HC}$ and $\mathrm{O}_{2}$ molar fractions.

Temperature slope is electronically regulated and $\mathrm{O}_{2}$ concentration is in a PID loop with upstream analyzer and mass flow meter. The GSV can be varied from $25000 \mathrm{~h}^{-1}$ to $150000 \mathrm{~h}^{-1}$ for a 0.4 -liter sample.

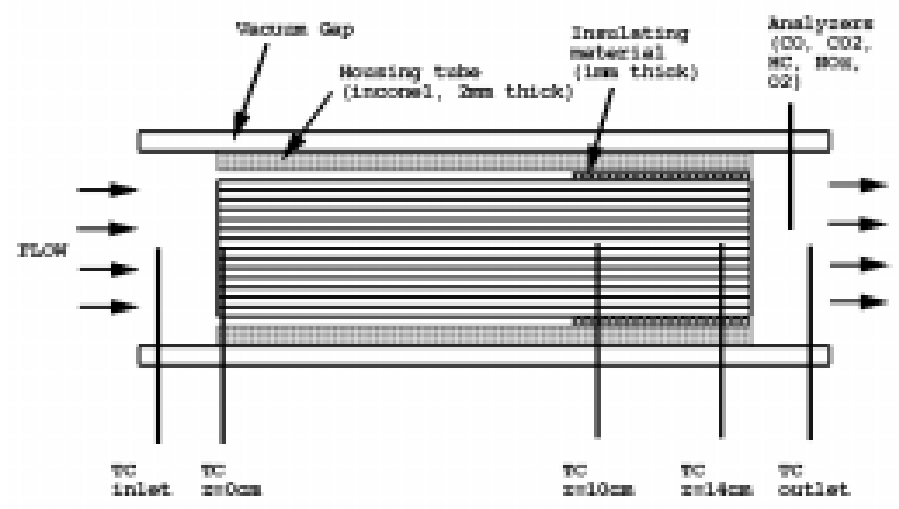

Figure 1 : Description of the experimental device (TC stands for ThermoCouple).

\section{SIC FILTER CHARACTERISTICS}

The ceramic substrate is made of SiC. The wall thickness is $0.33 \mathrm{~mm}$. The number of channel per square inch is 200 . The filter length is $152 \mathrm{~mm}$ and the filter diameter is $58 \mathrm{~mm}$ (filter volume $=0.4$ liter).

\section{TEST PROCEDURES}

Five identical filters have been prepared with different engine operating points and additive contents. Three filters have been loaded at a high load point characteristic of EUDC conditions $(2590 \mathrm{tr} / \mathrm{min}, 97 \mathrm{Nm})$ for three additive contents. These three filters will be referred to as EUDC 0 ppm, EUDC $25 \mathrm{ppm}$ and EUDC $50 \mathrm{ppm}$. Two filters have been loaded at a low load point characteristic of ECE conditions (2030 tr/min, $21.4 \mathrm{Nm}$ ). These two filters will be referred to as ECE 0 ppm and ECE $50 \mathrm{ppm}$.

These five filters have been regenerated with the same protocol. The GSV is kept constant $\left(\mathrm{GSV}=25000 \mathrm{~h}^{-1}\right)$. The gas temperature rises linearly from $100^{\circ} \mathrm{C}$ to $700^{\circ}$ in $1300 \mathrm{~s}$, then stays constant. The oxygen molar fraction decreases linearly from $18 \%$ to $4 \%$ then stays constant.

\section{PRESSURE DROP MODEL}

\section{DARCY'S LAW}

According to Sorenson et al. (1994), the total pressure drop across the filter is assumed to be the sum of the ceramic substrate pressure drop and the particulate layer pressure drop:

$$
\Delta P=\Delta P_{s}+\Delta P_{p}
$$

For the ceramic substrate and the particulate layer, the Darcy's law is assumed to be valid:

$$
\Delta P=\frac{\mu V E_{s}}{k_{s}}+\frac{\mu V E_{p}}{k_{p}}
$$

By using the concept of effective thickness of the particulate layer, the particulate layer pressure drop expression can be rewritten and finally:

$$
\Delta P=\frac{\mu V E_{s}}{k_{s}}+\frac{V m_{p} \mu}{A_{f}(\rho k)_{p}}
$$

or:

$$
\frac{\Delta P}{\mu V E_{s}}=\frac{1}{k_{s}}+\frac{m_{p}}{E_{s} A_{f}(\rho k)_{p}}
$$

\section{EXPERIMENTS}

The pressure drop curves are plotted in Figure 2. At 2000s, regeneration is over for all cases but the total pressure drop varies from 10 to 16 mbar. The filters are supposed to be clean. Thus, the total pressure drop only comes from the ceramic substrate. The most likely hypothesis is that the different samples used for these experiments are not perfectly identical. Work is ongoing to precisely determine the origin of this difference. 


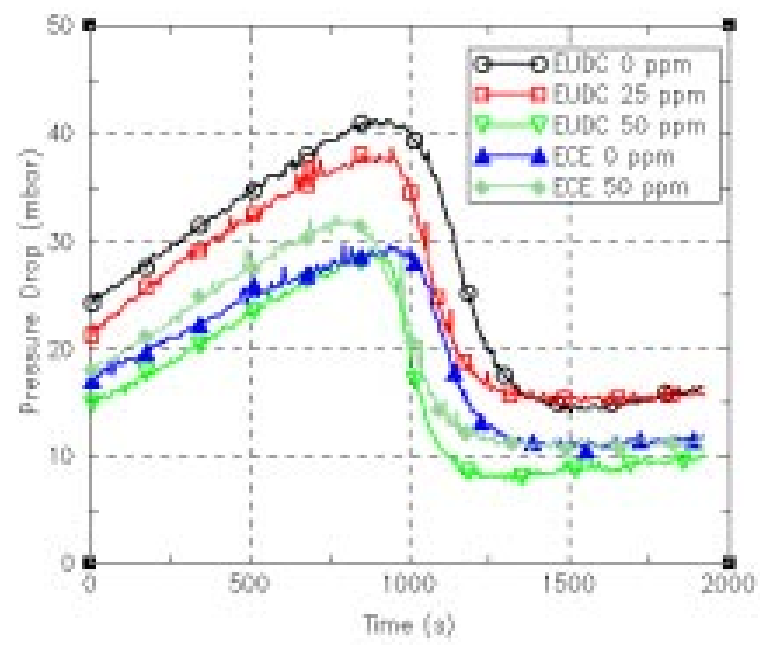

Figure 2: pressure curves for all cases

The soot mass in the filter $\mathrm{m}_{\mathrm{p}}$ as a function of time has been calculated from the measured $\mathrm{CO}, \mathrm{CO}_{2}$ and $\mathrm{HC}$ concentrations after the filter (see Figure 3). The Soluble Organic Fraction vaporization is clearly visible from 400s to 900s for all filters.

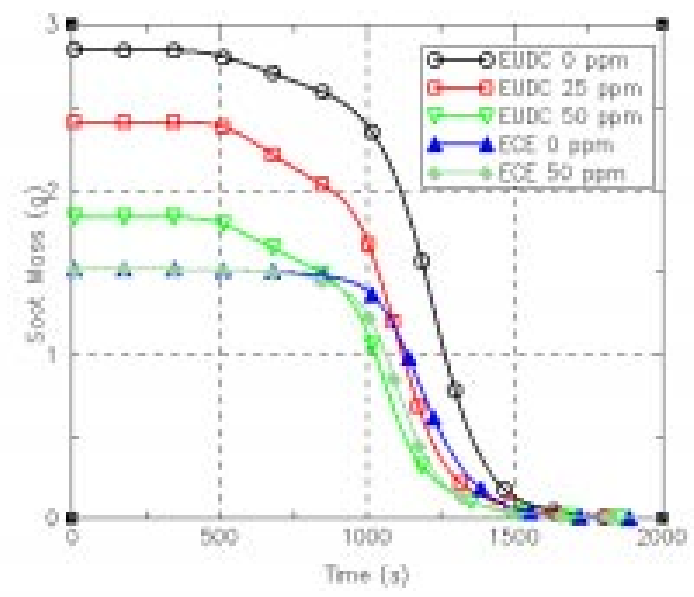

Figure 3: Soot mass in the filter during regeneration.

The initial soot mass are presented in Table 1.

\begin{tabular}{|l|l|}
\cline { 2 - 2 } \multicolumn{1}{c|}{} & Initial soot mass $(\mathrm{g})$ \\
\hline EUDC 0 ppm & 2.85 \\
\hline EUDC $25 \mathrm{ppm}$ & 2.41 \\
\hline EUDC 50 ppm & 1.83 \\
\hline ECE 0 ppm & 1.51 \\
\hline ECE $50 \mathrm{ppm}$ & 1.50 \\
\hline
\end{tabular}

Table 1
To assess the validity of Eq. (4) with constant values of $\mathrm{k}_{\mathrm{s}}$ and $(\rho \mathrm{k})_{\mathrm{p}}$, the ratio $\Delta \mathrm{P} /\left(\mu \mathrm{VE}_{\mathrm{s}}\right)$ has been plotted as a function of $m_{p}$ in Figure 4 . The curves are far from being straight lines as we would expect. This result is the motivation for the modeling that follows.

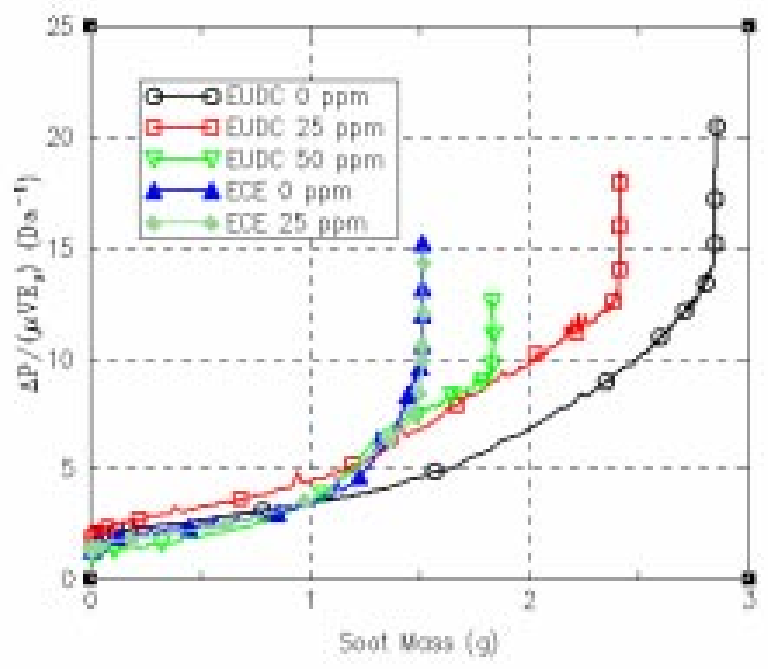

Figure 4: If wall and soot permeabilities are constant (see Eq. 4), the different curves in this plot should be straight lines.

\section{CERAMIC SUBSTRATE PERMEABILITY}

\section{Description of a standard loading curve}

Let us consider a standard loading experiment on the engine test bench for a constant operating condition. At the beginning of the loading, the filter is perfectly clean. Tan et al. (1996) noted that if the pressure drop across the filter is plotted as a function of the soot mass trapped in the filter (see Figure 5), one can clearly distinguish three regions:

- At the very beginning of the loading (Region 1), the pressure increases very rapidly. The particulates are trapped within the wall. The wall permeability changes from the clean filter permeability $\mathrm{k}_{\mathrm{s}-\mathrm{clean}}$ to the loaded filter permeability $\mathrm{k}_{\mathrm{s} \text {-loaded. }}$.

- After, this initial phase, the particulates start to accumulate on the wall surface (Region 2). This is a rather short transition phase.

- When the soot layer covers the whole wall surface, the particulates accumulate uniformly on the wall surface (Region 3). With region 3, the trap pressure drop is a linear function of the soot mass trapped in the filter. 


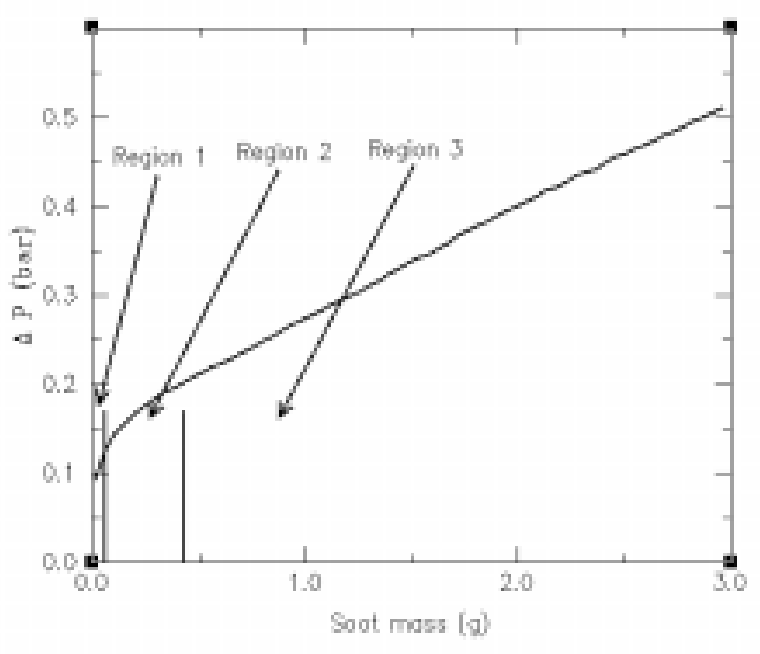

Figure 5: standard pressure drop for a loading experiment

\section{Simple modeling}

The modeling of regions 1 and 2 is very challenging and will not be tackled here. It will be assumed that the total duration of regions 1 and 2 is very small. Thus, the trap wall permeability jumps from $\mathrm{k}_{\mathrm{s} \text {-clean }}$ to $\mathrm{k}_{\mathrm{s} \text {-loaded }}$ at the start of the loading and then stays constant.

In the same way, during regeneration, the wall permeability is assumed to vary from $\mathrm{k}_{\mathrm{s} \text {-loaded }}$ to $\mathrm{k}_{\mathrm{s} \text {-clean }}$ when the total soot mass varies from the initial soot mass $m_{p i}$ to zero. The following simple modeling is finally retained:

$$
\frac{1}{k_{s}}=\frac{\left(m_{p} / m_{p i}\right)^{\sigma}}{k_{\text {s-loaded }}}+\frac{\left(1-m_{p} / m_{p i}\right)^{\sigma}}{k_{s-\text { clean }}}
$$

where $\sigma$ is a strictly positive coefficient. We will see above how the three constants $\mathrm{k}_{\mathrm{s} \text {-clean }}, \mathrm{k}_{\mathrm{s} \text {-loaded }}$ and $\sigma$ can be determined.

According to Sorenson et al. (1994), deviation from Darcy's law occurs for filtration velocities higher than $5 \mathrm{~cm} / \mathrm{s}$. For our experiments, the maximum filtration velocity is $4 \mathrm{~cm} / \mathrm{s}$. That is why Darcy's law has been used for this study.

\section{PARTICULATE LAYER PERMEABILITY}

The modern equations for flow through porous media date from 1856 when Darcy first studied water flowing through beds of sands:

$$
\Delta P=\frac{\mu V E}{k}
$$

This equation defines the constant $\mathrm{k}$, the permeability of the porous media. The permeability is conceived to be a property of the solid porous medium, independent of the porous medium, independent of the flowing fluid, and dependent upon such properties as porosity, pore size distribution, and surface area among others.

For higher velocities, it is found that another term must be added to Darcy's equation to accurately represent theoretical and experimental data:

$$
\Delta P=\frac{\mu V E}{k}+\rho B E V^{n}
$$

where $\mathrm{n}$ is a number close to 2 . With this equation and the new material constant $\mathrm{B}$, it is possible to predict the flow of fluids through porous media over a wide range of flow conditions for both liquids and gases. The constant B, according to theory should depend only on the porous medium and not on the fluid flowing through that medium.

\section{Slip flow}

Thus, Eq. (7) contains two constants, which depend only on the porous medium regardless of the fluid involved. The accumulated data indicate that this equation correlates a wide range of data for a variety of porous media. However, it was observed that the equation fails when applied to gas flow under certain conditions, e.g. larger values of $\mathrm{k}$ were obtained for gas flow than those obtained for liquid measurements and the $\mathrm{k}$ value varied for each gas. The difference was attributed to the slip conditions present when gas flows through the small passages of low porosity media. Even at atmospheric pressure the slip phenomenon is observed for pore sizes small enough, i.e. of the order of the mean free path of the gas. When the pore diameter $a$ is much larger that the mean free path $\lambda$ (in other words when the Knudsen number defined as $\mathrm{Kn}=\lambda / \mathrm{a}$ is much greater than unity), the fluid velocity at the wall can be considered as zero. On the contrary, when the mean free path is higher than the man pore diameter $(\mathrm{Kn}<1)$, one has to consider the non zero gas velocity at the wall.

The mean pore diameter for the wall is about $10 \mu \mathrm{m}$. The mean free path which can be calculated by the well know formula:

$$
\lambda=\frac{\mu}{P} \sqrt{\frac{\pi R T}{2 M}}
$$

is about $0.1 \mu \mathrm{m}$ for air at $1 \mathrm{bar}$ and $300 \mathrm{~K}$. The Knudsen number is lower or equal than $10^{-2}$. We will see later that when $\mathrm{Kn}<10^{-2}$, slip flow can be neglected.

For the soot layer, the mean pore diameter is assumed to be close to $0.1 \mu \mathrm{m}$ (see Salvat, 2000). The Knudsen number is close to unity and slip flow can not be neglected.

We will try to shortly review below the different studies which tried to predict how permeability is modified when slip flow is present. 
By considering the porous medium as a bundle of capillaries, Carman (1950) presented the following relationship for the permeability taken into account the slip flow:

$$
k / k_{K n=0}=1+\frac{6 \delta k}{k^{\prime}} K n
$$

where $\delta$, k and k' can be considered as constants.

Kuwabara (1959) determined the flow field permeability in a packed bed on the basis of the Stokes' approximation $(\mathrm{Kn}<<1)$ :

$$
k_{K n=0}=\frac{2 K a^{2}}{9 \varepsilon}
$$

where $\mathrm{K}$ is a function of the packing density $\varepsilon$ :

$$
K=1-9 / 5 \varepsilon^{1 / 3}+\varepsilon-1 / 5 \varepsilon^{2}
$$

Lee et al. (1978) modified the analysis of Kuwabara (1959) to take into account the slip flow. The calculated influence of $\mathrm{Kn}$ on Eq. (10) is:

$$
k / k_{K n=0}=\frac{\left(1+\frac{3 C m K n G}{K}\right)}{(1+2 C m K n)}
$$

where the momentum accommodation coefficient $\mathrm{C}_{\mathrm{m}}$ is of order unity and where $\mathrm{G}$ is a function of the packing density:

$$
G=1-6 / 5 \varepsilon^{1 / 3}+1 / 5 \varepsilon^{2}
$$

Lee et al. (1978) determined that for a porous medium with a porosity of 0.6 , it is legitimate to neglect the influence of the Knudsen number when $\mathrm{CmKn}$ is lower that $10^{-2}$.

Later, Pulkrabek and Ibele (1987) experimentally studied the influence of temperature on the permeability of a porous medium. They obtained the following formula:

$$
k / k_{K n=0}=1+C_{1} \lambda
$$

where $\mathrm{C}_{1}$ is a function of T. $\mathrm{C}_{1}$ varies from 1.4 at $300 \mathrm{~K}$ to 0.4 at $1500 \mathrm{~K}$ then seems to level off.

The relationship experimentally found by Pulkrabek and Ibele (1987) is different from the one from Carman (1950) because $C_{1}$ is not a true constant.

\section{Contribution of this work}

It seems that there is no consensus on the right formula which should be used to predict the pressure drop for nonzero Knudsen numbers. It turned out that for our experimental results, it was sufficient to assume that $(\rho \mathrm{k})_{\mathrm{p}}$ was proportional to $\lambda$ to obtain a good agreement:

$$
(\rho k)_{p}=c \frac{\lambda}{\lambda_{\text {ref }}}
$$

The pressure used in the formula for the mean free path is taken to be the average pressure in the filter. For our experiments, we obtained:

$$
c=0.55 \mathrm{Da} \mathrm{kg} / \mathrm{m}^{3}
$$

There are at least two indications for the validity of this approach.

Pattas et al. (1997) have estimated $(\rho \mathrm{k})_{\mathrm{p}}$ as function of trap outlet temperature for different filter loading levels (test vehicle with standard EX-80 filter, 100ppm Cerium in fuel). They obtained values of $(\rho \mathrm{k})_{\mathrm{p}}$ in the range 0.3 to $1.0 \mathrm{Da}$ $\mathrm{kg} / \mathrm{m}^{3}$. This range is coherent with Eq. (16). They also remarked that $(\rho k)_{p}$ is a clearly increasing function of $T$. Again, this is coherent with Eq. (15). These authors attribute this temperature dependence to Soluble Organic Fraction vaporization. We thus suggest another explanation based on the linearity between $(\rho \mathrm{k})_{\mathrm{p}}$ and $\lambda$. Experiments are ongoing at PSA to study pressure drop for soot without Soluble Organic Fraction.

Sorenson et al. (1994) have studied the influence of the pressure drop on $(\rho \mathrm{k})_{\mathrm{p}}$. They have noted that $(\rho \mathrm{k})_{\mathrm{p}}$ is a decreasing function of $\Delta \mathrm{p}$. They assumed that it was a consequence of the compression of the soot layer when $\Delta \mathrm{p}$ increases. With our model, the product $(\rho \mathrm{k})_{\mathrm{p}}$ is also a decreasing function of $\Delta \mathrm{p}$ but the reason has nothing to do with the compression of the soot layer. It is because the mean free path is proportional to the reciprocal of the mean pressure in the soot layer. Sorenson's explanation and ours are likely to be valid at the same time. This point also needs further studies.

\section{MODEL VALIDATION}

Adding the two models described above, we finally obtain the following relationship:

$$
\frac{\Delta P}{\mu V E_{s}}=\frac{\left(m_{p} / m_{p i}\right)^{\sigma}}{k_{s-\text { loaded }}}+\frac{\left(1-m_{p} / m_{p i}\right)^{\sigma}}{k_{s-\text { clean }}}+\frac{m_{p} \lambda_{\text {ref }}}{c E_{s} A_{f} \lambda}
$$

Eq. (17) contains four adjustable constants: $\mathrm{c}, \mathrm{k}_{\text {s-loaded }}, \mathrm{k}_{\mathrm{s} \text {-clean }}$ and $\sigma$. To determine the different parameters of the above relationship, the ratio $\mathrm{Ry}=\Delta \mathrm{P} /\left(\mu \mathrm{VE}_{\mathrm{s}}\right)$ is plotted as a function of $R x=m_{p} \lambda_{\text {ref }} /\left(E_{s} A_{f} \lambda\right)$ in Figure 6 for the EUDC 0 ppm case. 


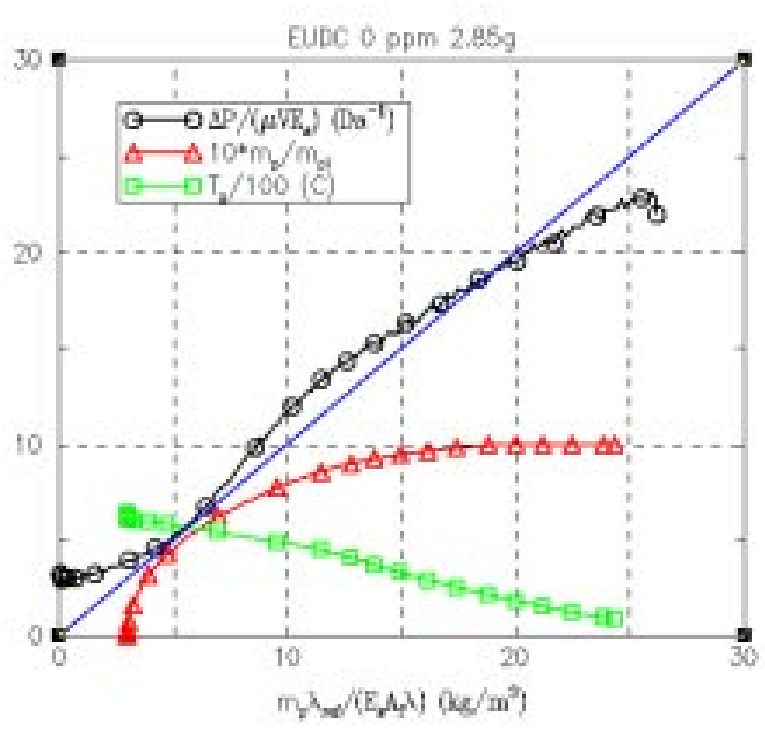

Figure 6: base plot to determine the parameters of the pressure drop model.

At the start of the experiment, the temperature is low. The mean free path is low and the soot mass is equal to the initial soot mass. In other words, $\mathrm{Rx}$ is maximum. As long as the temperature has not reached about $500^{\circ} \mathrm{C}$, one can assume that the ceramic substrate permeability $\mathrm{k}_{\mathrm{s}}$ is constant. That is Ry should be a linear function of Rx. This is in agreement with our experimental data. The slope of this line enables us to obtain

$$
c=0.55 \mathrm{Da} \mathrm{kg} / \mathrm{m}^{3}
$$

and the intercept gives us

$$
k_{\text {s-loaded }}=0.12 \mathrm{Da}
$$

When regeneration is almost over, $\mathrm{Rx}$ is close to zero and the total pressure drop is solely due to the clean wall permeability. The $\mathrm{Ry}$ value for $\mathrm{Rx}=0$ gives us

$$
k_{\text {s-clean }}=0.33 \mathrm{Da}
$$

Finally the value of $\alpha$ is determined to give a good agreement between measurements and calculations when oxidation is at its higher rate. We found

$$
\sigma=2.2
$$

With these parameters, we present the comparison between measurements and simulations in Figure 7 for the EUDC 0 ppm case.

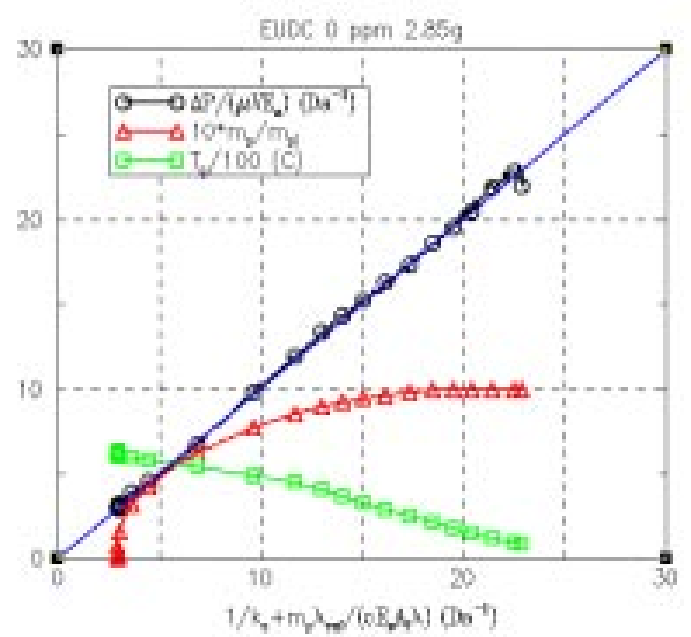

Figure 7: final plot when all parameters have been adjusted.

With the assumption that these constants could be used for the other cases, the comparison between simulations and experiments is presented for all cases in Figure 8. We know that this is only a first approximation. However, the global agreement can be considered as correct.

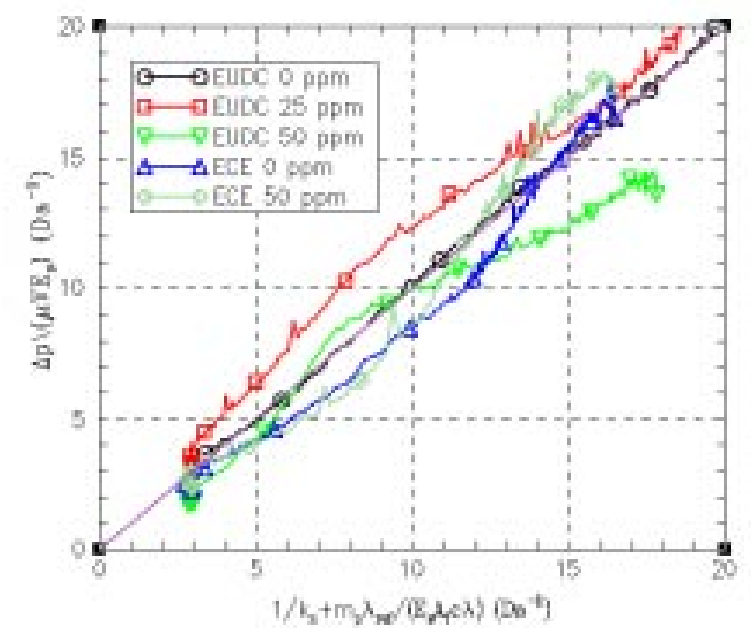

Figure 8: comparison between measurements and simulations for all pressure curves.

Experiments are ongoing to determine how $\mathrm{c}, \mathrm{k}_{\text {s-loaded }}, \mathrm{k}_{\text {s-clean }}$ and $\sigma$ depend on the engine operating conditions (load, speed and additive content). However for this study, the simple model described above has been considered as sufficient. 


\section{CATALYTIC REGENERATION MODEL}

Mathematical models of the regeneration process without catalytic aids have been developed to understand the phenomenon and optimize filter systems since early 80's (Bissett, 1983). Mathematical models for the catalytic regeneration process with fuel additives are currently based on the oxygen transfer theory. According to this theory, the fuel additive contained in the accumulated soot chemically stores and exchanges oxygen atoms with the surrounding carbon and exhaust gas. Based on this assumption, we have previously developed a zero-dimensional (Koltsakis and Stamatelos, 1996) and a 1-dimensional (Koltsakis and Stamatelos, 1997) model of the catalytic filter regeneration.

Zero-dimensional models assume identical flow conditions of the exhaust gas flowing through the filter wall along the channel. This assumption is quite realistic for high flow rate operation. However, operation with low flow rates is interesting especially during regeneration with the engine idling, which is a possible filter failure scenario. In the latter case, significant variations in flow and temperature conditions are expected along the channel, which should be predicted by an extended 1D model. This model is briefly outlined below.

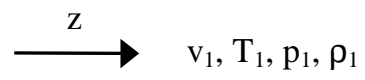

Inlet channel

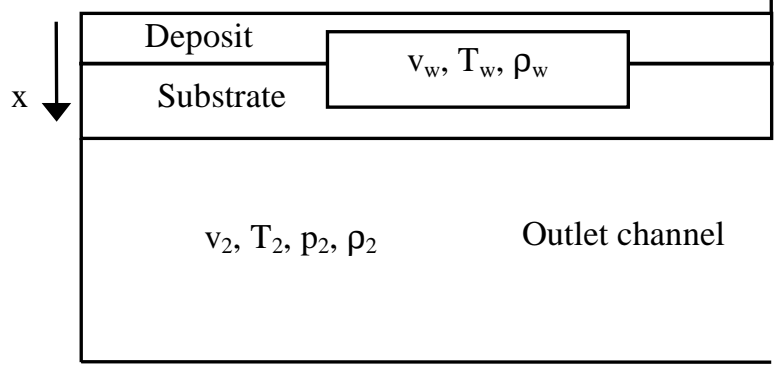

Figure 9: Schematic of the model inlet and outlet channel of a wall flow honeycomb filter

Figure 9 presents a schematic diagram of the monolith inlet and outlet channels with the substrate wall and the soot deposit layer. The inter-phase heat transfer within the wall is so large that the gas and solid temperatures may be taken equal except in a very thin boundary layer at the interface with the inlet channel. The governing equations for the conservation of mass, momentum and energy in the flowing exhaust gas are given below:

\section{Conservation of mass of channel gas}

In the balance equation for mass conservation, the mass flowing into or from the elementary control volume through the filter wall should be taken into account:

$$
\frac{\partial}{\partial z}\left(\rho_{i} v_{i}\right)=(-1)^{i}(4 / D) \rho_{w} v_{w}
$$

\section{Conservation of z-component of momentum of channel} gas

$$
\frac{\partial p_{i}}{\partial z}+\frac{\partial}{\partial z}\left(\rho_{i} v_{i}^{2}\right)=-\alpha_{1} \mu v_{i} / D^{2}
$$

The r.h.s. term of Eq. (23) represents the pressure losses in the axial flow direction $\mathrm{z}$ caused by the viscous drag forces. Since the mass flow passing through the wall is only a small fraction of the axial flow, the velocity profile should be close to that observed in flows in closed channels. Thus, the relation used to compute the pressure loss is the one used for laminar flows in square ducts.

\section{Conservation of energy of channel gas}

In the formulation of the energy balance of the channel gas, we take into account the convective heat exchange with the channel wall, as well as the enthalpy flowing into or from the elementary control volume through the filter wall. For the inlet channel the gas leaves the control volume at temperature $\mathrm{T}_{1}$ :

$$
\begin{aligned}
& \left.C_{p, g}\left|D^{2} \rho_{1} v_{1} T_{1}\right|_{z+\Delta z}-\left.D^{2} \rho_{1} v_{1} T_{1}\right|_{z}+\left.4 D \Delta z \rho_{w} v_{w} T_{1}\right|_{z}\right\rfloor \\
& =h_{1} 4 D \Delta z\left(T_{w}-T_{1}\right)
\end{aligned}
$$

combining Eq. (22) and Eq. (24), we obtain:

$$
\left.C_{p, g}\left(\rho_{1} v_{1}\right)\right|_{z+\Delta z} \frac{\partial T}{\partial z}=h_{1}(4 / D)\left(T_{w}-T_{1}\right)
$$

On the other hand, for the outlet channel, the gas enters the control volume at temperature $\mathrm{T}_{\mathrm{w}}$ :

$$
\begin{aligned}
& \left.C_{p, g}\left|D^{2} \rho_{2} v_{2} T_{2}\right|_{z+\Delta z}-\left.D^{2} \rho_{2} v_{2} T_{2}\right|_{z}-\left.4 D \Delta z \rho_{w} v_{w} T_{w}\right|_{z}\right\rfloor \\
& =h_{2} 4 D \Delta z\left(T_{w}-T_{2}\right)
\end{aligned}
$$

Analogously, combining Eqs. (22) and (26):

$$
\begin{aligned}
\left.C_{p, g}\left(\rho_{2} v_{2}\right)\right|_{z} \frac{\partial T}{\partial z} & = \\
& \quad\left(h_{2}+C_{p, g} \rho_{w} v_{w}\right)(4 / D)\left(T_{w}-T_{2}\right)
\end{aligned}
$$

\section{Soot combustion and CO selectivity}

Typical diesel particulates consist mainly of a carbonaceous core (soot formed during combustion), adsorbed compounds such as unburned and partially oxygenated hydrocarbons, sulphates (due to the oxidation of the sulphur contained in the fuel) and metal oxides (Vuk et al., 1976). The incomplete carbon oxidation, is assumed to be described by the following reaction:

$$
\mathrm{C}+\alpha \mathrm{O}_{2} \rightarrow 2(\alpha-0.5) \mathrm{CO}_{2}+2(1-\alpha) \mathrm{CO}
$$


where $\alpha$ is an index of the completeness of the reaction taking values from 0.5 to 1 . For modeling purposes, the parameter $\alpha$ should, be estimated beforehand by means of exhaust gas analysis. Measured values for soot reported in the literature range between 0.55 and 0.9 (de Soete, 1987; Aoki et al., 1993).

$\Delta \mathrm{H}$ indicates a combined reaction enthalpy resulting from the complete and incomplete oxidation of carbon, which is linked to $\alpha$ according to the relation:

$$
\Delta H=2(\alpha-0.5) \Delta H_{(i)}+2(1-\alpha) \Delta H_{(i i)}
$$

The overall rate of soot combustion without catalytic aids is assumed to follow a simple Arrhenius type expression:

$$
k_{1}=k T e^{-E / R T}
$$

For the apparent activation energy E appearing in Eq. (30), several values have been proposed ranging from 80.000 to $160.000 \mathrm{~J} / \mathrm{mol}$ (Bissett and Shadman 1985, Pauli et al., 1984, Hoffmann and Ma, 1990). Engine experiments and theoretical investigations (Lepperhoff and Kroon, 1984; Koltsakis and Stamatelos, 1996b) indicate that a value of $150.000 \mathrm{~J} / \mathrm{mol}$ satisfactorily represents regeneration reaction behaviour.

\section{Kinetics and mechanism of additive action}

According to Koltsakis and Stamatelos (1996), we use the following definitions:

$$
\xi=\frac{\text { moles of metal oxides present in soot }}{\text { carbon moles present in soot }}
$$

The fraction $\xi$, which is the catalyst concentration in the soot, is a function of the metal additive concentration in the fuel as well as the engine soot emissions produced during the filter loading operation.

Metal additive oxides can in principle be present in the deposit layer in both the lower and the higher oxidation state. We define:

$$
\psi=\frac{\text { "higher oxidation state" metal oxy. present in soot }}{\text { total metal oxides present in soot }}
$$

During catalytic regeneration the oxidation state of the metal oxides may be changed by reacting either with oxygen of the exhaust gas or with the carbon atoms of the deposit layer. Thus, if we assume that the metal additive Me forms oxides with its 1 - and 2-valent states, the following redox reactions take place:

$$
\begin{aligned}
& \mathrm{C}+4 \alpha_{\mathrm{cat}} \mathrm{MeO} \\
& \rightarrow 2 \alpha_{\text {cat }} \mathrm{Me}_{2} \mathrm{O}+2\left(\alpha_{\text {cat }}-0.5\right) \mathrm{CO}_{2}+2\left(1-\alpha_{\text {cat }} \mathrm{CO}\right) \\
& \mathrm{Me}_{2} \mathrm{O}+\frac{1}{2} \mathrm{O}_{2} \rightarrow 2 \mathrm{MeO}
\end{aligned}
$$

where $\alpha_{\text {cat }}$ is here an index for the completeness of the catalytic carbon oxidation, taking values from 0.5 to 1 .

These reactions combine with reaction (28) to complete the reaction scheme for catalytic soot oxidation. They represent a continuous oxidation/reduction process of the metal oxides present in the deposit soot layer. The rate of the reduction reaction (33) is assumed to follow an Arrhenius-type temperature dependence according to the following equation:

$$
R_{\text {red }}=k_{\text {red }} \psi e^{-E_{\text {red }} / R T}
$$

The rate of the heterogeneous oxidation reaction (34) is expected to be proportional to the oxygen content of the exhaust gas, as well as to the availability of "lower oxidation state" metal oxides, which is expressed by 1- $\psi$. An Arrhenius-type temperature dependence is also assumed here. The reaction rate for (34) is then:

$$
R_{o x}=k_{o x} \cdot\left[O_{2}\right] \cdot(1-\psi) \cdot e^{-E_{o x} / R T}
$$

The total rate of change of $\psi$ may then be written as:

$$
\frac{d \psi}{d t}=R_{o x}-R_{\text {red }}
$$

Considering the stoichiometry of the reduction reaction the mass balance equation for the deposit layer, assuming that the deposit is consumed in a shrinking mode, gives (Koltsakis and Stamatelos, 1996a):

$$
\begin{aligned}
& \rho_{p} \frac{d w_{p}}{d t}= \\
& -\left(\frac{M_{c}}{M_{O_{2}}}\right) \rho_{\mathrm{w}} v_{w} y \frac{1}{\alpha}\left(1-\exp \left(-\frac{S_{p} k_{1}\left(T_{w}\right) w_{p}}{v_{w}} \alpha\right)\right) \\
& -\frac{1}{2 \alpha_{c a t}} \rho_{\mathrm{p}} w_{p} \xi R_{\text {red }}
\end{aligned}
$$

\section{Energy balance in the wall}

The energy balance equation for the wall should take into account the contributions of convective heat transfer from the channel flow and from the flow through the wall, the heat released by the exothermic soot combustion as well as the conductive heat transfer along the channel wall:

$$
\begin{aligned}
& \frac{\partial}{\partial t}\left(\rho_{p} C_{p, p} T_{w}+\rho_{s} C_{p, s} T_{w}\right)= \\
& h_{1}\left(T_{1}-T_{w}\right)+h_{2}\left(T_{2}-T_{w}\right) \\
& +\rho_{w} v_{w} C_{p, g}\left(T_{1}-T_{w}\right)+H_{\text {react }}+H_{\text {cond }}
\end{aligned}
$$

From the equation for the consumption rate of the deposit (38), we can compute the heat released by the overall reaction expressed per unit time and area:

$$
\begin{aligned}
& H_{\text {react }}=\left(-\frac{\Delta H}{M_{O_{2}}}\right) \rho_{w} v_{w} y \frac{1}{\alpha}\left[1-\exp \left(-\frac{S_{p} k_{1}\left(T_{w}\right) w_{p}}{v_{w}}\right)\right] \\
& -\frac{1}{4 \alpha_{\text {cat }} M_{c}} \rho_{w} v_{w} \Delta H \xi R_{\text {red }}
\end{aligned}
$$

The contribution of heat conduction is:

$$
H_{\text {cond }}=-\lambda_{p} \frac{\partial}{\partial z}\left(w_{p} \frac{\partial T_{w}}{\partial z}\right)-\lambda_{s} w_{s} \frac{\partial^{2} T_{w}}{\partial z^{2}}
$$

\section{Pressure drop across the deposit layer and ceramic wall}

In order to compute the flow field in the filter channel, a correlation for the pressure drop through the soot layer and 
the filter wall is needed. According to the analysis presented in the previous section, we employ Eq. 17.

\section{Initial and boundary conditions}

The initial temperature, soot loading and catalyst concentration along the channel wall are provided as initial conditions for the model. The boundary conditions, which need to be defined, include the exhaust gas temperature, flow rate and oxygen content as functions of time as well as the pressure at the filter exit.

\section{Solution procedure}

The governing equations presented above are solved numerically with finite difference techniques using an iterative procedure in the spatial direction controlled by the requirements of zero axial velocity at the end of the inlet channel and atmospheric pressure at the end of the exit channel. Time marching was effected with a 4th order Runge-Kutta technique. More details on the solution procedure can be found in Koltsakis and Stamatelos (1997).

\section{MODEL VALIDATION}

For $\mathrm{SiC}$ filters the initiation of soot regeneration is observed close to the completion of $\mathrm{HC}$ desorption. This allowed us to decouple the two phenomena and consider only soot oxidation, neglecting HC desorption. From a numerical point of view, the problem was fixed by using an initial soot mass equal to the soot mass without Soluble Organic Fraction. The influence of this approximation on the pressure drop modeling has been neglected.

\section{Regeneration without additive for EUDC case}

With the pressure drop model described above, the simulation of the regeneration curves has been tackled.

The oxidation rate contains two parameters: the activation energy $\mathrm{E}$ and the pre-exponential factor $\mathrm{k}$. Standard values have been used (see Koltsakis and Stamatelos, 1997):

$$
\left\{\begin{array}{l}
E=150 \mathrm{~kJ} / \mathrm{mole} \\
k=1 \mathrm{~m} /(\mathrm{sK})
\end{array}\right.
$$

and there was no need to modify these constants to obtain the agreement shown in Figure 10 for EUDC 0 ppm. Soot combustion is predicted to start at $850 \mathrm{~s}$ as in the experimental data.

\section{$\underline{\text { Regeneration with additive for EUDC cases }}$}

The kinetic constants linked to catalytic oxidation have been adjusted with the EUDC 25 ppm case:

$$
\left\{\begin{array}{l}
E_{\text {red }}=E_{o x}=100 \mathrm{~kJ} / \mathrm{mole} \\
k_{\text {red }}=k_{o x} / 5=6 \times 10^{4} s^{-1}
\end{array}\right.
$$

The results are presented in Figure 10. The presence of Cerium produces an earlier start of combustion $(750 \mathrm{~s}$ versus $850 \mathrm{~s})$.

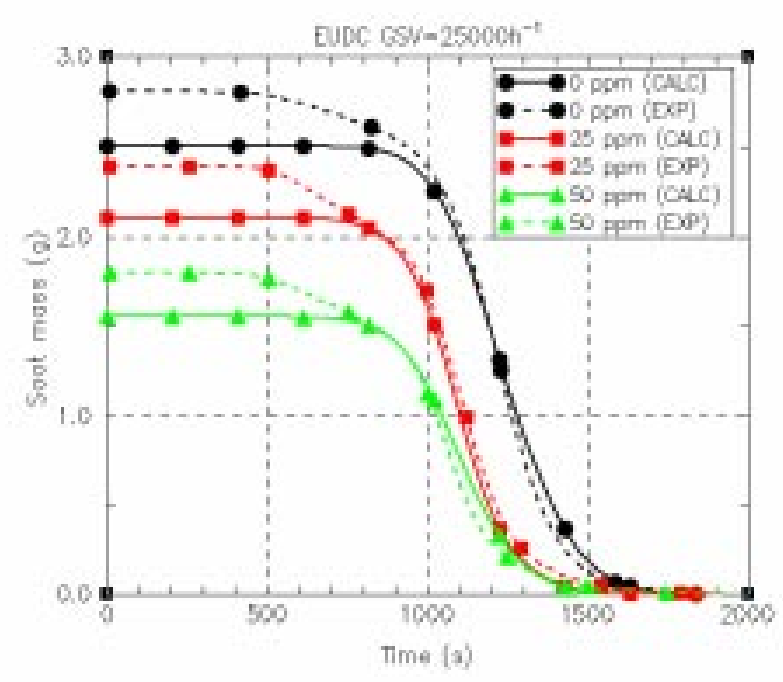

Figure 10: comparison between experiments and simulations for all EUDC regenerations.

Without modifying these parameters, the EUDC $50 \mathrm{ppm}$ case has been simulated (see Figure 10). The agreement is correct. It can be noted that increasing the additive content from $25 \mathrm{ppm}$ to $50 \mathrm{ppm}$ does not modify the start of oxidation. This trend is clear both with experiments and simulations.

\section{Regeneration with and without additive for ECE cases}

Without modifying the parameters determined above, the ECE cases have been simulated. The results are presented in Figure 11. The predicted regeneration for the $0 \mathrm{ppm}$ case is slightly more rapid than the measured one. On the other hand the predicted regeneration for the $50 \mathrm{ppm}$ case is a bit slower than the measurement. Given that the pressure drop model and combustion model parameters were left untouched, it can be considered as a good test of the validity of the approach. 


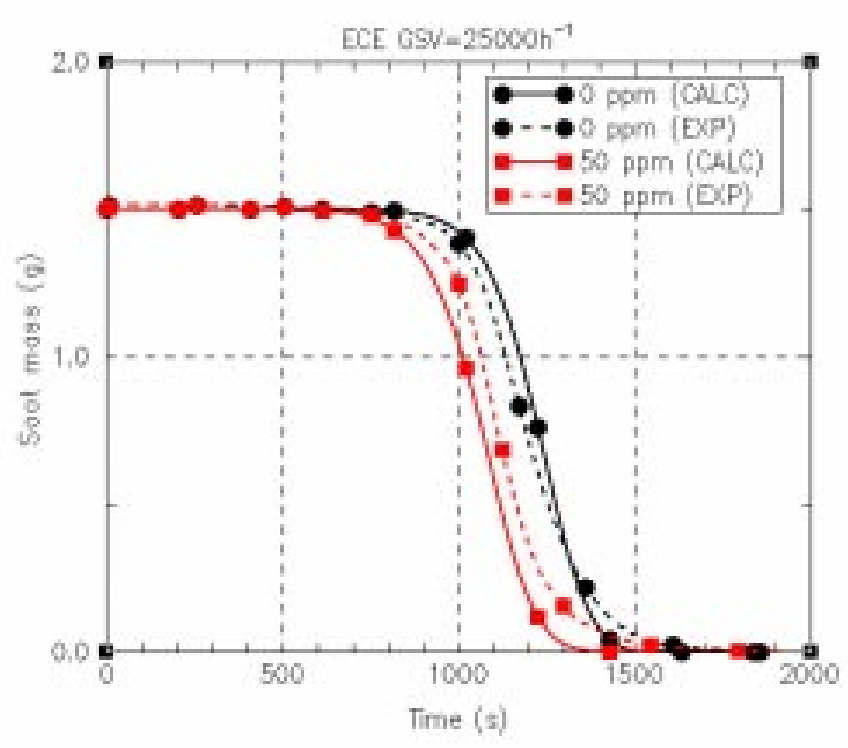

Figure 11: comparison between experiments and simulations for all ECE regenerations

\section{CONCLUSIONS}

The standard pressure drop modeling which assumes that wall and soot permeabilities are constant has proved unable to explain our experimental data. To remedy this problem, an extended pressure drop correlation has been developed. The product of the soot layer permeability and the soot layer density has been assumed to be proportional to the gaseous mean free path. Although the first results are encouraging, the influence of the soot composition on the pressure drop has not been clarified. Experiments are ongoing to study the influence of the soot composition on the pressure drop. With this data, it will be possible to assess the validity of the suggested correlation.

With this new pressure drop correlation, regenerations with and without additive have been simulated. The catalytic oxidation model can be fitted to give the right influence of the additive content on regeneration.

\section{Nomenclature}

\section{$\mathrm{A}_{\mathrm{f}} \quad$ Filtration area}

a Mean pore diameter

B Constant in the non-linear Darcy's equation

$\mathrm{C}_{1} \quad$ Function of temperature only

$\mathrm{Cm}$ Momentum accommodation coefficient

$\mathrm{C}_{\mathrm{p}, \mathrm{g}} \quad$ Specific heat capacity of exhaust gas, $1090 \mathrm{~J} / \mathrm{kgK}$

$\mathrm{C}_{\mathrm{p}, \mathrm{p}} \quad$ Specific heat capacity of particulate layer, $1510 \mathrm{~J} / \mathrm{kgK}$

$\mathrm{C}_{\mathrm{p}, \mathrm{s}} \quad$ specific heat capacity of ceramic substrate, $1120 \mathrm{~J} / \mathrm{kgK}$

D hydraulic diameter of channel
E apparent activation energy of soot oxidation, $\mathrm{J} / \mathrm{mol}$

$\mathrm{E}_{\mathrm{OX}}$ activation energy for metal additive oxidation, $\mathrm{J} / \mathrm{mol}$

$\mathrm{E}_{\text {red }}$ activation energy for metal additive reduction, $\mathrm{J} / \mathrm{mol}$

$\mathrm{E}_{\mathrm{p}} \quad$ Particulate layer thickness, $\mathrm{m}$

$\mathrm{E}_{\mathrm{s}} \quad$ Ceramic substrate thickness, $\mathrm{m}$

G Function of the packing density only

GSV Gas Space Velocity, $\mathrm{h}^{-1}$

$\mathrm{H}$ heat convection coefficient, $\mathrm{W} / \mathrm{m}^{2} \mathrm{~K}$

$\mathrm{H}_{\text {react }}$ reaction heat release, $\mathrm{W} / \mathrm{m}^{2}$

$\mathrm{H}_{\text {cond }}$ conductive heat flux, $\mathrm{W} / \mathrm{m}^{2}$

$\Delta \mathrm{H} \quad$ "combined" reaction enthalpy of soot oxidation, $\mathrm{J} / \mathrm{mol}$

$\Delta \mathrm{H}_{\text {(i) }}$ specific heat of $\mathrm{CO}_{2}$ formation, $3.61 \times 10^{5} \mathrm{~J} / \mathrm{mol}$

$\Delta \mathrm{H}$ (ii) specific heat of $\mathrm{CO}$ formation, $0.90 \times 10^{5} \mathrm{~J} / \mathrm{mol}$

$\mathrm{k}_{1}$ rate coefficient for the soot combustion reaction, $\mathrm{m} / \mathrm{s}$

$\mathrm{k} \quad$ collisions frequency factor, $1.0 \mathrm{~m} / \mathrm{sK}$

$\mathrm{k}_{\mathrm{ox}} \quad$ rate coefficient for fuel additive oxidation, $\mathrm{s}^{-1}$

$\mathrm{k}_{\mathrm{s}} \quad$ permeability of particulate deposit layer, $\mathrm{m}^{2}$

$\mathrm{k}_{\text {red }}$ rate coefficient for fuel additive reduction, $\mathrm{s}^{-1}$

$\mathrm{k}_{\mathrm{s}} \quad$ permeability of ceramic substrate, $\mathrm{m}^{2}$

$\mathrm{k}_{\mathrm{s} \text {-clean }}$ Clean ceramic substrate permeability, $\mathrm{m}^{2}$

$\mathrm{k}_{\mathrm{s} \text {-loaded }}$ Load ceramic substrate permeability, $\mathrm{m}^{2}$

$\mathrm{M}_{\mathrm{O}_{2}}$ molecular weight of exhaust gas, $29 \times 10^{-3} \mathrm{~kg} / \mathrm{mol}$

$\mathrm{M}_{\mathrm{c}} \quad$ atomic weight of deposit, $12 \times 10^{-3} \mathrm{~kg} / \mathrm{mol}$

$\mathrm{m}_{\mathrm{p}} \quad$ Soot mass in the filter, $\mathrm{kg}$

$\mathrm{m}_{\mathrm{pi}} \quad$ Initial soot mass in the filter, $\mathrm{kg}$

$\mathrm{P} \quad$ Exhaust gas pressure, $\mathrm{Pa}$

$\mathrm{R} \quad$ Perfect gas constant, $8.31 \mathrm{~m}^{3} \mathrm{~Pa} / \mathrm{mol} \mathrm{K}$

$\mathrm{Rx} \quad$ Variable defined by $\mathrm{Ry}=\Delta \mathrm{P} /\left(\mu \mathrm{VE}_{\mathrm{s}}\right)$

Ry Variable defined by $\mathrm{Rx}=\mathrm{m}_{\mathrm{p}} \lambda_{\text {ref }} /\left(\mathrm{E}_{\mathrm{s}} \mathrm{A}_{\mathrm{f}} \lambda\right)$

$\mathrm{S}_{\mathrm{p}} \quad$ specific area of deposit layer, $5.5 \times 10^{7} \mathrm{~m}^{-1}$

$\mathrm{T}$ temperature, $\mathrm{K}$

$\mathrm{t}$ time, $\mathrm{s}$

v velocity, $\mathrm{m} / \mathrm{s}$

$\mathrm{V} \quad$ Mean filtration velocity, $\mathrm{m} / \mathrm{s}$

$\mathrm{w}_{\mathrm{p}} \quad$ thickness of the particulate layer, $\mathrm{m}$

$\mathrm{w}_{\mathrm{s}} \quad$ Channel wall thickness, $\mathrm{m}$

$\mathrm{x}$ distance, $\mathrm{m}$

$y \quad$ oxygen mole fraction of the exhaust gas, [-]

$\mathrm{z} \quad$ axial distance, $\mathrm{m}$

\section{Greek letters}

$\alpha \quad$ Index for the completeness of thermal soot oxidation, $[-]$

$\alpha_{1} \quad$ Constant in channel pressure drop correlation, 28.45

$\delta \quad$ Constant, [-]

$\varepsilon \quad$ Packing density, [-]

$\Delta \mathrm{P} \quad$ Trap back-pressure, $\mathrm{Pa}$

$\Delta \mathrm{P}_{\mathrm{s}} \quad$ Ceramic substrate pressure drop, $\mathrm{Pa}$

$\Delta \mathrm{P}_{\mathrm{p}} \quad$ Particulate layer pressure drop, $\mathrm{Pa}$

$\lambda \quad$ Gas mean free path, $\mathrm{m}$

$\lambda_{\text {ref }} \quad$ Reference gas mean free path $(\mathrm{P}=1$ bar, $\mathrm{T}=300 \mathrm{~K})$

$\lambda_{\mathrm{p}} \quad$ Particulate thermal conductivity, $2.1 \mathrm{~W} / \mathrm{mK}$ 
$\lambda_{\mathrm{s}} \quad$ Substrate thermal conductivity, $0.85 \mathrm{~W} / \mathrm{mK}$

$\mu \quad$ Exhaust gas viscosity

$\rho \quad$ Exhaust gas density, $\mathrm{kg} / \mathrm{m}^{3}$

$\rho_{\mathrm{p}} \quad$ Bulk density of the deposit layer, $140 \mathrm{~kg} / \mathrm{m}^{3}$

$\rho_{\mathrm{s}} \quad$ Bulk density of porous ceramic, $1400 \mathrm{~kg} / \mathrm{m}^{3}$

$\sigma \quad$ Contant, [-]

$\psi \quad$ Defined in Eq. 32, [-]

$\xi \quad$ Defined in Eq. 31, [-]

\section{$\underline{\text { Subscripts }}$}

b Initial condition

cat Catalytic

$\mathrm{i}=1,2 \quad$ Inlet, outlet channel

$\mathrm{p} \quad$ Particulate layer

s Substrate

\section{Abbreviations}

GSV Gas Space Velocity

\section{References}

Aoki H., Asano A., Kurazono K., Kobashi K., Sami H., "Numerical Simulation Model for the Regeneration Process of a Wall- Flow Monolith Diesel Particulate Filter", SAE paper 930364, 1993.

Bissett E., Mathematical Modeling of the Thermal regeneration of a Wall-Flow Monolith Diesel particulate Filter", Chem. Eng. Sci., 39 (7/8) 1233, 1984.

Bissett E., Shadman F., Thermal Regeneration of Diesel-Particulate Monolithic Filters, AlChE Journal (Vol 31,No 5), p. 753, May 1985.

Carman, P. C., Diffusion and flow of gases and vapours through micropores. I. Slip flow and molecular streaming, Proc. R. Soc. London, A203, 55-74, 1950.

De Soete G., Combustion Catalytique des Suies Formees en Phase Gazeuse a Partir de Diesel-oils. Institut Francais du Petrole Techniques d'applications Energetiques, Rapport IFP No 35378, Juin 1987.

Hoffmann U., Ma J., Study on Regeneration of Diesel Particulate Filter Using a Laboratory Reactor, Chemical Engineering Technology,13, p.251-258, 1990.

Koltsakis G. C. and Stamatelos A. M., Modeling Catalytic Regeneration of Wall-Flow Particulate Filters. Industrial \& Engineering Chemistry Research, 35, pp. 2-13, 1996a.

Koltsakis G. C. and Stamatelos A. M., Modeling Thermal Regeneration of Wall-Flow Diesel Particulate Traps. AIChE Journal, Vol. 42, No. 6, 1996 b.

Koltsakis G. C. and Stamatelos A. M., Modes of Catalytic Regeneration in Diesel Particulate Filters, Ind. Eng. Chem. Res. 1997, 36, 4155-4165.

Kuwabara, S., The forces experienced by randomly distributed parallel circular cylinders or spheres in a viscous flow at small Reynolds numbers, Journal of the physical society of Japan, Vol. 14, No 4, 527-532, 1959.
Lee, K. W., Reed, L. D. and Gieseke, J. A., pressure drop across packed bed in the low Knudsen number regime, J. Aerosol Sci., Vol. 9, pp. 557-565, 1978.

Lepperhoff, G., Kroon, G., Abgasnachbehandlung I (Abschlussbericht), FVV-Heft 352, 1984.

Pattas K. N., Stamatelos A. M., Koltsakis G. C, Kandylas I. P., Mustel W., Computer Aided Engineering in the Design of Catalytically Assisted Trap Systems. SAE paper 970472, 1997.

Pulkrabek, W. W. and Ibele, E. I., The effect of temperature on the permeability of a porous material, Int. J. Heat Mass Transfer, Vol. 30, No 6, pp 1103-1109, 1987.

Salvat, O., Marez, P. and Belot, G., 2000, Passenger Car Serial Application of a Particulate Filter System on a Common Rail Direct Injection Diesel Engine, SAE 2000.

Sorenson S., Hoj J. Stobbe P. Flow Characteristics of SiC Diesel Particulate Filter Materials. SAE paper 940236, 1994. Tan, J.C., Opris, C.N., Baumgard, K.J. and J.H. Johnson: A Study of the Regeneration Process in Diesel Particulate Traps Using a Copper Fuel Additive. SAE paper 960136, 1996.

Vuk C. T., Jones M. A., Johnson J. H., The measurement and analysis of the physical character of diesel particulate emissions. SAE paper 760131, 1976. 